\title{
Cultural Adaptation, Reliability and Validation of the Arabic Örebro Musculoskeletal Pain Questionnaire in Patients With Low Back Pain
}

Muhammad Z. Alrwaily ( $\square$ muhammad.alrwaily@hsc.wvu.edu )

West Virginia University https://orcid.org/0000-0001-9406-6788

Fahad Alanazi

Al-Jouf University College of Applied Medical Sciences

\section{Research}

Keywords: Low back pain, Örebro Musculoskeletal Pain Questionnaire, Arabic, Cultural adaptation, validation

Posted Date: September 29th, 2021

DOl: https://doi.org/10.21203/rs.3.rs-876349/v1

License: (c) (1) This work is licensed under a Creative Commons Attribution 4.0 International License.

Read Full License

Version of Record: A version of this preprint was published at Journal of Pain Research on February 1st, 2023. See the published version at https://doi.org/10.2147/JPR.S375202. 


\section{Abstract}

BACKGROUND: The Örebro Musculoskeletal Pain Questionnaire (ÖMPQ) assesses the psychosocial factors in people with complaints of musculoskeletal disorders and predict those likely to develop persistent symptoms.

OBJECTIVE: To culturally-adapt and assess the validity of the ÖMPQ in an Arabic population with low back pain (LBP).

METHODS: The ÖMPQ was translated into Arabic. Eighty-four patients completed the questionnaires at baseline, 2 days later, and 3 months follow-up. The test-retest reliability, interclass correlation coefficient (ICC), specific agreement, predictive validity and content validity were assessed.

RESULTS: For construct validity, the Arabic ÖMPQ (A-ÖMPQ) had a moderate $(r \geq 0.3,<0.5)$ to high ( $r \geq$ $0.5)$ correlation with pain, disability, fear-avoidance and catastrophizing questionnaires. The test-rest reliability showed was high $\mathrm{ICC}_{2,1}=0.92(95 \% \mathrm{Cl}$ : .83 - .96). The A-ÖMPQ score at baseline can significantly predict disability at 3 months $F(1,82)=33.87, p<.01 ; \mathrm{R}^{2}=.29$.

CONCLUSIONS: The translation of the A-ÖMPQ into Arabic was successful. The A-ÖMPQ showed very good psychometric properties, and thus can be used to evaluate the psychosocial factors amongst patients with LBP.

\section{Introduction}

Low back pain (LBP) is a common health problem around the globe. It affects $40 \%$ of individuals at some point in their life (1), and is a leading cause of disability and healthcare financial burden (2). In the Arab world (22 countries), LBP-related disability has reached levels similar to those of the Western world (3). Among high-income Arab countries (6 countries), LBP is ranked the 4 th reason for years lost due to disability (3). In Saudi Arabia, LBP may be affecting $53-79 \%$ of people (4), and it is also considered the 3rd leading cause of disability after motor vehicle accidents and diabetes (2). Due to the rapid rise of such conditions, the Ministry of Health in Saudi Arabia through the World Health Organization Regional Office has called for programs and policies to reduce the burden of chronic diseases in all countries in the Eastern Mediterranean (3).

Given the huge burden of LBP in Saudi Arabia, it is important that primary care clinicians are able to identify patients at risk of developing persistent symptoms and make early referrals to physical therapy. In Saudi Arabia, patients with spinal disorders represent $28.1 \%$ of referrals to physical therapy clinics, of which $53.1 \%$ are due to LBP (5). From the time of referral, it seems that patients who took 16 days or more till their 1st physical therapy session were less likely to adhere to the treatment plan and more likely to develop persistent symptoms (6). In a Saudi population, development of persistent LBP symptoms has been associated with physical factors such as pain intensity, physical activity and body mass index; as well as psychosocial factors such as fear-avoidance, unhelpful beliefs, poor expectation about recovery, 
anxiety and depression (7). These factors, despite cultural differences, are similar to those reported in the Western world (8). It is recognized that early assessment of these factors will help clinicians identify patients at risk of persistent disability and direct them to the optimal path of care early (9).

One tool that assesses patients' risk of developing persistent disability is the Örebro Musculoskeletal Pain Questionnaire (ÖMPQ) (10). The ÖMPQ uses multiple domains to predict disability including physical factors such as pain intensity and location; and psychological factors such as fear-avoidance, unhelpful beliefs, poor recovery expectations, catastrophizing, anxiety and depression. The ÖMPQ consists of 25 items of which 21 are scored. Each scored item can take a value from 1-10, for a total score of 210 . A score of 105 or less indicates low risk of developing persistent disability (accuracy of 95\%), a score of more than 105 and less than 130 indicates moderate risk of developing persistent disability, and score of 130 or more indicates high risk of persistent disability (accuracy of $86 \%$ ) at 6 months $(10,11)$. The ÖMPQ has been shown reliable and valid (10), and can aid primary care providers in evaluating psychosocial factors and prescribe appropriate intervention.

Since its release in 1998, the ÖMPQ English version has been translated, culturally adapted and validated into several languages and populations (12-15). However, there is no translation, cultural adaptation, or validation in an Arabic population thus far. Arabic is one of the most commonly used languages in the world, and considered one of the 6 official languages by the United Nations (16). Therefore, the aim of this study is to culturally adapt and evaluate the psychometric properties of the ÖMPQ in a primary care clinic in Saudi Arabia.

\section{Materials And Methods}

\section{Translation of the Arabic ÖMPQ}

Based on Beaton et al. guidelines (17), we conducted a multistep translation of the ÖMPQ. The first step was a forward translation where two translators whose native language was Arabic and proficient in English translated the ÖMPQ into Arabic. The two Arabic translations were synthesized into a single form. The second step was backward translation where two translators whose native language was English and proficient in Arabic translated the synthesized Arabic ÖMPQ back into English. In the third step, an expert committee was formed to examine all the translations from the forward, backward and synthesized versions, and create a prefinal version of the Arabic ÖMPQ. The expert committee consisted of 2 researchers and 3 clinicians familiar with the Saudi culture who worked to resolve any discrepancy in the translation process and ensure that the prefinal version is appropriate for use in the Saudi culture. The prefinal version was distributed on 23 consecutive patients at a primary care out-patient facility to examine the comprehension of each item and resolve any misinterpretation. Since there were no issues with the prefinal version, the expert committee decided to use it as the final version.

\section{Validation of the Arabic ÖMPQ}

\section{Design}


We used the final version of the Arabic ÖMPQ to test the psychometric properties in a primary care outpatient clinic in a hospital in Saudi Arabia. We asked participants to complete questionnaires at baseline, 2 days later, and then 3 months follow-up. We powered the study for reliability and validity using the minimum required number of participants of 50 (18). To account for dropout, we aimed to increase the number of participants to 80 . This study was approved by the Office of Research Integrity and Compliance in the authors' hospital.

\section{Participants}

The inclusion period was between March 2018 and February 2019. Participants were included if they were 18 years or older, had acute or chronic LBP, and were fluent in Arabic. Participants were excluded if they had fracture, cancer, inflammatory conditions, infections, severe neurological deficits, post-surgical, psychiatric disorder, or pregnant. Participants who consented to participate were asked to complete the questionnaires; and then received usual care.

\section{Measurements}

Session 1 (at Baseline): Participants completed a demographics form, the Arabic versions of the ÖMPQ, Oswestry Disability Index (ODI) (19), Numeric Pain Rating Scale (NPRS), Fear-Avoidance Behavior Questionnaire-Physical Activity and Work scales (FABQ-PA; FABQ-W) (20), and the Pain Catastrophizing Scale (PCS) (21).

Session 2 ( 2 days later): Each participant was asked if their symptoms changed from last session? Also, participants were asked to complete the Arabic NPRS and ÖMPQ again to investigate the retest reliability.

Session 3 (3 months later): Participants were asked to complete the Arabic versions of ODI and NPRS. Also, they were asked if their symptoms changed from last sessions?

\section{Statistical Analysis}

All statistical analysis was done using SPSS 25 (Armonk, NY: IBM Corp.) We used descriptive statistics to describe participants' characteristics and the prevalence of the 3 risk profiles (low, medium, high) on the Arabic ÖMPQ.

For construct validity, we analyzed the characteristics of pain, disability, fear-avoidance behavior and catastrophizing across the ÖMPQ risk profile to determine the discriminant validity. We also calculated Pearson's correlation coeffıcient between specific ÖMPQ items and their respective questionnaires according to each item domain. We used Bier et al. (22), a priori expectations that there would be moderate $(r \geq 0.3,<0.5)$ to high $(r \geq 0.5)$ correlation between pain items 9,10 and 11 with the NPRS; catastrophizing items $12,13,14$ and 15 with PCS; fear-avoidance of physical activity items 18 and 19 with FABQ-PA scale; and fear-avoidance of work item 20 with FABQ-W scale; disability items 6, 20, 21, 22, 23, 24 and 25 with ODI. We expected a low correlation $r<0.3$ between items 8 (perception of work demands), 16 (expectation to return to work) and 17 (work satisfaction) with FABQ-W scale. 
For reliability, we assessed the agreement between two measurements in a group of participants whose condition remained stable between session 1 and session 2 . We considered a stable condition when the participant responded "yes" to the question "Have your symptoms changed from the last session?"; and the NPRS score did not change more than \pm 1 point. We calculated the interclass coefficient $\left(\operatorname{ICC}_{2,1}\right)$ and the specific agreement. The ICC 2,1 was interpreted as follows $\leq 0$ = poor; $.01-.02$ = slight; $.21-.4$ = fair; $.41-.60=$ moderate; $.61-.80=$ substantial; and $.81-1=$ perfect. We also calculated specific agreement using a $3 \times 3$ table similar to that reported Bier et al. (22).

For predictive validity, we calculated simple linear regression to determine whether ÖMPQ at baseline can predict ODI at 3 months. Also, we calculated the relative risk ratio (RR) for the medium risk group and high risk group relative to the low risk group. For RR, we considered persistent disability when the ODI score at 3 months remained above the median of the baseline score.

For content validity, we investigated floor and ceiling effect by calculating the percentage of participants scoring 0 (floor) or 210 (ceiling). If the percentage was $>15 \%$, then floor or ceiling effect was present.

\section{Results}

We recruited 84 participants whose demographics are listed in Table 1. At baseline, $53.6 \%$ of participants were in the low risk group, $38.1 \%$ were in the medium risk group and $8.3 \%$ were in the high risk group. 
Table 1

Demographics and clinical characteristics at baseline.

\begin{tabular}{|lll|}
\hline & N & $\%$ \\
\hline Age, y, mean (SD) & $33.1(10.6)$ & \\
\hline Sex & & \\
\hline Male & 54 & 64.3 \\
\hline Female & 30 & 35.7 \\
\hline Education & & \\
\hline Secondary school & 24 & 28.6 \\
\hline College degree & 54 & 64.3 \\
\hline Higher education & 6 & 7.1 \\
\hline Employment & & \\
\hline Student & 8 & 6.5 \\
\hline Employed & 51 & \\
\hline Unemployed & 18 & \\
\hline Retired & 7 & \\
\hline Clinical characteristics & & \\
\hline Symptoms duration, m, mean (SD) & $58.2(66.4)$ \\
\hline NPRS mean (SD) & $5.3(2.3)$ & \\
\hline ODI mean (SD) & $29.6(19.5)$ & \\
\hline FABQ-PA mean (SD) & $12.1(5.7)$ & \\
\hline
\end{tabular}

Data are reported as numbers and percentages unless stated otherwise.

$\mathrm{SD}=$ standard deviation

NPRS = numeric pain rating scale

$\mathrm{ODI}=$ Oswestry Disability Index

FABQ-PA = Fear-avoidance behavior questionnaire Physical Activity

FABQ-W = Fear-avoidance behavior questionnaire Work

PCS $=$ Pain catastrophizing Scale

A-ÖMPQ = Arabic Örebro Musculoskeletal Pain Questionnaire 


\begin{tabular}{|lc|}
\hline \multicolumn{3}{|l|}{ N } & $\%$ \\
\hline FABQ-W mean (SD) & $16.4(9.9)$ \\
\hline PCS mean (SD) & $21.6(11.5)$ \\
\hline A-ÖMPQ mean (SD) & $98.3(25.9)$ \\
\hline Risk profile & \\
\hline Low & 45 \\
\hline Medium & 32 \\
\hline High & 7 \\
\hline Data are reported as numbers and percentages unless stated otherwise. \\
\hline SD = standard deviation \\
\hline NPRS = numeric pain rating scale \\
\hline ODI = Oswestry Disability Index \\
\hline FABQ-PA = Fear-avoidance behavior questionnaire Physical Activity \\
\hline FABQ-W = Fear-avoidance behavior questionnaire Work \\
\hline PCS = Pain catastrophizing Scale \\
\hline A-ÖMPQ = Arabic Örebro Musculoskeletal Pain Questionnaire \\
\hline
\end{tabular}

For construct validity, it appeared that for each increase in risk profile on the ÖMPQ, there was an increase in clinical characteristics of pain, disability, fear-avoidance and catastrophizing (Table 2). Also, the Pearson's $r$ correlation between each ÖMPQ item and its corresponding clinical outcome is reported in Table 3. There was moderate to high correlation between the pain items 9,10 and 11 with the NPRS. There was a low correlation between the catastrophizing item 12 with PCS; however, moderate to high correlation between the catastrophizing items 13,14 and 15 with PCS. There was a high correlation between the fear-avoidance (physical activity) items 18 and 19 with FABQ-PA scale. There was a high correlation between the perception of work demands item 8 with FABQ-W scale; however, there was a low correlation between the fear-avoidance (work) item 20, expectation to return to work item 16 and work satisfaction item 17 with FABQ-W scale. There was moderate to high correlation between the disability items $6,21,22,23,24$ and 25 with ODI. 
Table 2

Clinical characteristic across risk profiles of A-ÖMPQ

\begin{tabular}{|llll|}
\hline & $\begin{array}{l}\text { Low } \\
(\leq 105)\end{array}$ & $\begin{array}{l}\text { Medium } \\
(>105 \text { and }<130)\end{array}$ & $\begin{array}{l}\text { High } \\
(\geq 130)\end{array}$ \\
\hline A-ÖMPQ, N (\%) & $45(53.6)$ & $32(38.1)$ & $7(8.3)$ \\
\hline Pain (NPRS) & $4.5(2.2)$ & $5.7(1.8)$ & $8.6(1.9)$ \\
\hline Disability (ODI) & $20.7(14.4)$ & $37.1(17.8)$ & $53.4(23.0)$ \\
\hline Fear-avoidance (FABQ) & $24.0(13.1)$ & $32.0(10.0)$ & $41.9(11.7)$ \\
\hline Catastrophizing (PCS) & $15.3(9.8)$ & $27.3(8.5)$ & $35.6(8.3)$ \\
\hline SD = standard deviation & & & \\
\hline NPRS = numeric pain rating scale & & \\
\hline ODI = Oswestry Disability & & \\
\hline FABdex $=$ Fear-avoidance behavior questionnaire & \\
\hline PCS = Pain catastrophizing Scale & \\
\hline A-ÖMPQ = Arabic Örebro Musculoskeletal Pain Questionnaire \\
\hline
\end{tabular}


Table 3

Pearson's correlation between each A-ÖMPQ item and outcome measures

\begin{tabular}{|lllll|}
\hline A-ÖMPQ item & Outcome Measure & Pearson's $r$ & Expected a priori \\
\hline 1 & - & - & - & - \\
\hline 2 & - & - & - & - \\
\hline 3 & - & - & - & - \\
\hline 4 & - & - & - & - \\
\hline 5 & - & - & - & - \\
\hline 6 & ODI & .38 & Moderate & Yes \\
\hline 7 & - & - & - & - \\
\hline 8 & FABQ - W & .51 & High & No \\
\hline 9 & NPRS & .47 & Moderate & Yes \\
\hline 10 & NPRS & .30 & Moderate & Yes \\
\hline 11 & NPRS & .36 & Moderate & Yes \\
\hline 12 & PCS & .11 & Low & No \\
\hline 13 & PCS & .39 & Moderate & Yes \\
\hline 14 & PCS & .39 & Moderate & Yes \\
\hline 15 & PCS & .59 & High & Yes \\
\hline 16 & FABQ - W & .16 & Low & Yes \\
\hline 17 & FABQ - W & -.09 & Low & Yes \\
\hline 18 & FABQ - PA & .52 & High & Yes \\
\hline 19 & FABQ - PA & .53 & High & Yes \\
\hline
\end{tabular}

$(-)=$ Item not applicable for analysis

NPRS $=$ Numeric Pain Rating Scale

$\mathrm{ODI}=$ Oswestry Disability Index

FABQ-PA = Fear-avoidance behavior questionnaire Physical Activity

FABQ-W = Fear-avoidance behavior questionnaire Work

PCS $=$ Pain catastrophizing Scale

A-ÖMPQ = Arabic Örebro Musculoskeletal Pain Questionnaire 


\begin{tabular}{|lllll|}
\hline A-ÖMPQ item & Outcome Measure & \multicolumn{2}{l}{ Pearson's $r$} & Expected a priori \\
\hline 20 & FABQ - W & .23 & Low & No \\
21 & ODI & .26 & Low & No \\
\hline 22 & ODI & .39 & Moderate & Yes \\
\hline 23 & ODI & .54 & High & Yes \\
\hline 24 & ODI & .44 & Moderate & Yes \\
\hline 25 & ODI & .45 & Moderate & Yes \\
\hline$(-)=$ Item not applicable for analysis & & & \\
\hline NPRS = Numeric Pain Rating Scale & & & \\
\hline ODI = Oswestry Disability Index & & \\
\hline FABQ-PA = Fear-avoidance behavior questionnaire Physical Activity \\
\hline FABQ-W = Fear-avoidance behavior questionnaire Work
\end{tabular}

For reliability, there was 45 participants whose status was determined to be stable, so they were included in the assessment of reliability. The $\mathrm{ICC}_{2,1}$ for the ÖMPQ was $.92(95 \% \mathrm{Cl}$ : .83 - .96). The specific agreement for the low risk group was $78.26 \%$, medium risk group was $71.79 \%$ and high risk group was $40 \%$ (Table 4). 
Table 4

Specific agreement between session 1 (baseline) and session 2 (48 hours later)

\begin{tabular}{|c|c|c|c|c|}
\hline & \multicolumn{3}{|c|}{$\begin{array}{l}\text { Session } 2 \\
\text { (2 days later) }\end{array}$} \\
\hline & & Low & Medium & High \\
\hline \multirow{3}{*}{$\begin{array}{l}\text { Session } 1 \\
\text { (Baseline) }\end{array}$} & Low & $18(\mathrm{~A})$ & $3(\mathrm{~B})$ & $0(\mathrm{C})$ \\
\hline & Medium & $6(D)$ & $14(E)$ & $0(\mathrm{~F})$ \\
\hline & High & $1(\mathrm{G})$ & $2(\mathrm{H})$ & $1(\mathrm{I})$ \\
\hline \multicolumn{5}{|c|}{ Low risk: $A /(A+(B+C+D+G) / 2)=18 / 23=78.26 \%$} \\
\hline \multicolumn{5}{|c|}{ Medium risk: $E /(E+(B+H+D+F) / 2)=14 / 19.5=71.79 \%$} \\
\hline \multicolumn{5}{|c|}{ High risk: $\mathrm{I} /(\mathrm{I}+(\mathrm{C}+\mathrm{F}+\mathrm{G}+\mathrm{H}) / 2)=1 / 2.5=40 \%$} \\
\hline
\end{tabular}

For the predictive validity, the simple linear regression shows that the ÖMPQ at baseline can significantly predict the ODI at 3 months $F(1,82)=33.87, p<.01$; with $\mathrm{R}^{2}$ of .29 . To calculate the RR, persistent disability at 3 months was set at or above the ODI baseline median score of 26 . The RR for the medium risk group compared with the low risk group was 1.9 (95\% Cl: 1.2-3.3). The RR for the high risk group compared with the low risk group was 2.3 (95\% Cl: .7-7.7).

For content validity, we investigated the floor and ceiling and there was no participant scoring 0 (floor effect) or 210 (ceiling effect).

\section{Discussion}

This study translated the ÖMPQ into Arabic and tested its reliability and validity. The translation of the ÖMPQ was appropriate as the expert committee ensured that the items are reader-friendly and close to Saudi Arabian culture and dialect. The Arabic ÖMPQ was understood well by patients in primary care clinics and we did not have any issues with items comprehension.

For the construct validation, it was important that the Arabic ÖMPQ can distinguish between patients with LBP who have low, medium, or high psychosocial status. We did not use receiver operating curve because we did not want to dichotomize the scores. To that end, this study showed that for each increase in psychosocial risk status on the Arabic ÖMPQ, there was an associated increase in the outcome measures of pain, disability, fear-avoidance and catastrophizing. This suggested that the Arabic ÖMPQ had good discriminant validity between low, medium and high risk groups.

Since the ÖMPQ had multiple constructs such as pain, disability, fear-avoidance, and catastrophizing, we investigated if these constructs are correlated with their respective outcome measures. Similar to 
correlations found in other studies (12-15), the constructs within the Arabic ÖMPQ showed significant moderate to high correlations with their respective outcome measures. The correlations were as expected a priori and suggest that the Arabic ÖMPQ is capable of capturing various levels of psychological factors.

There were 3 items in the Arabic ÖMPQ that correlated different than what was set a priori: item 8 (perception of work demands), item 12 (pain control), and item 20 (fear-avoidance of work). Item 8 was expected to have low correlation $(r<.3)$ with FABQ-W but it was found to have high correlation $(r \geq .5)$, suggesting better than expected correlation between perception of work demands and fear-avoidance of work. Item 12 was expected to have at least moderate correlation with pain catastrophizing but the correlation was low, suggesting that item 12 should be considered in light of other pain catastrophizing items 13,14 and 15 . Item 20 was expected to have at least moderate correlation with the FABQ-W but had low correlation, suggesting that item 8 and 20 should be evaluated together when assessing work demands or fear avoidance of work.

The reliability of the Arabic ÖMPQ was tested between session 1 (baseline) and session 2 (2 days later). Since LBP could change rapidly, the 2-day time interval was considered long enough to reduce recall bias, and short enough to prevent substantial change in status. The reliability was tested with $\mathrm{ICC}_{2,1}$ showed perfect reliability, which was similar to the levels reported in in the Norwegian and Brazilian translated versions $(12,15)$. We also investigated specific agreements for each risk status between session 1 and 2 . It appeared that for the low and medium risk status, there was very good agreement. However, for the high risk status, there was a fair agreement likely due to smaller number of participants in this category.

We investigated the predictive validity of the Arabic ÖMPQ in two ways. One, we used simple linear regression to investigate if the ÖMPQ score at baseline is predictive of the disability score on ODI at 3 months. The simple regression showed that the ÖMPQ at baseline can predict disability at 3 months and can explain $29 \%$ of the variability in disability at 3 months. Two, we used RR to determine if being in one risk category increases the chances of developing persistent disability. Participants in the medium risk profile have 1.9 higher chance of developing persistent disability compared to the low risk group. Also, participants in the high risk group have 2.3 higher chances of developing disability compared to the low risk group. The confidence interval of medium risk and high risk groups were overlapped, also the confidence interval of high risk group crossed 1. This could be due to patient receiving treatment "usual care", or due to smaller number of participants in the high risk group.

We recognized that the original ÖMPQ had been modified to address several limitations such as inconsistent wording, reduced practicality, and lack of independent validation (23). So, just like in Gabel et al. (23), we carefully selected wording of the questions that are related to symptom duration, activity, function variables, and psychosocial constructs. Also, we attempted to divide the Arabic ÖMPQ into themed sections for ease of response and grading (Appendix 1). However, unlike Gabel et al. (23), we did not renumber the questions nor did we modify the construct "pain" to "pain/problem" or reduce the number of body regions. We did so because we were hoping that the Arabic ÖMPQ would be used for conditions other than LBP. 
There were several reasons to choose the ÖMPQ for cultural adaptation and validation in Arabic population. At the time of the study, there were not validated psychosocial screening tools for Arabic people with musculoskeletal pain. Also, the ÖMPQ may be more useful clinically as the responses to its items are scaled from 0 to 10 as opposed to dichotomized with "yes" and "no", which can help clinicians probe on constructs that need further assessment (24). Finally, the ÖMPQ is commonly recommended by clinical practice guidelines of $\operatorname{LBP}(25,26)$.

Several limitations of this study can be identified. We only validated the Arabic ÖMPQ patients with LBP, and we do not know if it generalizes to other musculoskeletal cases. We only used the level disability as a predicted variable of poor outcome, and we do not know if other clinical outcomes can be predicted with Arabic ÖMPQ. Future research should investigate whether the Arabic ÖMPQ improves the delivery of healthcare and reduces its cost.

\section{Conclusion}

The Arabic ÖMPQ should be used in primary care and physical therapy clinics to assist in early screening of patients with LBP with elevated psychosocial profile, and determine those at risk of developing poor treatment outcome. The Arabic ÖMPQ has been translated and validated for patient with LBP in primary care clinics in a Saudi Arabian population. The translation was appropriate and the psychometric properties of reproducibility, and construct, content and predictive validity are appropriate for screening psychosocial factors in patients with LBP.

\section{Abbreviations}

FABQ-PA: Fear-Avoidance Behavior Questionnaire-Physical Activity

FABQ-W: Fear-Avoidance Behavior Questionnaire-Work

ICC: Interclass Correlation Coeficient

LBP: low back pain

NPRS: Numeric Pain Rating Scale

ODI: Oswestry Diability Index

ÖMPQ: Örebro Musculoskeletal Pain Questionnaire

PCS: Pain Catastrophizing Scale

RR: Risk Ratio

\section{Declarations}




\section{Ethical Approval and Consent of Participants}

This study was approved by the Office of Research Integrity and Compliance at West Virginian University School of Medicine \# 1710818925

\section{Consent of Publication}

Not Applicable

\section{Availability of Supporting Data}

The datasets used during the current study are available from the corresponding author upon request.

\section{Competing Interest}

The authors declare that they have no competing interests.

\section{Funding}

The authors received no funding for this study.

\section{Author's Contributions}

MA and FA equally participated in designing the study, collecting, analyzing, and interpreting the patient data, as well as writing the manuscript. All authors read and approved the final manuscript.

\section{Acknowledgement}

We would like to thank Samuel Lee Harris, Sarah Tarabichi, Abdulrahman Alruwaili, Asheg Alanazi, and Smar Alothman for their contribution in the translation and data collection.

\section{References}

1. Hoy D, Bain C, Williams G, March L, Brooks P, Blyth F, et al. A systematic review of the global prevalence of low back pain. Arthritis and rheumatism. 2012;64(6):2028-37.

2. DALYs GBD, Collaborators H, Murray CJ, Barber RM, Foreman KJ, Abbasoglu Ozgoren A, et al. Global, regional, and national disability-adjusted life years (DALYs) for 306 diseases and injuries and healthy life expectancy (HALE) for 188 countries, 1990-2013: quantifying the epidemiological transition. Lancet. 2015;386(10009):2145-91.

3. Mokdad AH, Jaber S, Aziz MI, AlBuhairan F, AlGhaithi A, AlHamad NM, et al. The state of health in the Arab world, 1990-2010: an analysis of the burden of diseases, injuries, and risk factors. Lancet. 2014;383(9914):309-20.

4. Awaji M. Epidemiology of low back pain in Saudi Arabia. Journal of Advances in Medical and Pharmaceutical Sciences. 2016;6(4):1-9. 
5. Alshami AM. Prevalence of spinal disorders and their relationships with age and gender. Saudi medical journal. 2015;36(6):725-30.

6. Al-Eisa E. Indicators of adherence to physiotherapy attendance among Saudi female patients with mechanical low back pain: a clinical audit. BMC musculoskeletal disorders. 2010;11:124.

7. Alamam D, Moloney N, Leaver A, Alsobayel HI, Mackey MG. Pain Intensity and Fear Avoidance Explain Disability Related to Chronic Low Back Pain in a Saudi Arabian Population. Spine. 9000; Publish Ahead of Print.

8. Ramond A, Bouton C, Richard I, Roquelaure Y, Baufreton C, Legrand E, et al. Psychosocial risk factors for chronic low back pain in primary care--a systematic review. Family practice. 2011;28(1):12-21.

9. Hill JC, Whitehurst DG, Lewis M, Bryan S, Dunn KM, Foster NE, et al. Comparison of stratified primary care management for low back pain with current best practice (STarT Back): a randomised controlled trial. Lancet. 2011;378(9802):1560-71.

10. Linton SJ, Boersma K. Early Identification of Patients at Risk of Developing a Persistent Back Problem: The Predictive Validity of The Örebro Musculoskeletal Pain Questionnaire. The Clinical journal of pain. 2003;19(2):80-6.

11. Brown G. The Orebro musculoskeletal pain questionnaire. Occupational medicine (Oxford, England). 2008;58(6):447-8.

12. Grotle M, Vollestad NK, Brox Jl. Screening for yellow flags in first-time acute low back pain: reliability and validity of a Norwegian version of the Acute Low Back Pain Screening Questionnaire. The Clinical journal of pain. 2006;22(5):458-67.

13. Nonclercq O, Berquin A. Predicting chronicity in acute back pain: validation of a French translation of the Orebro Musculoskeletal Pain Screening Questionnaire. Annals of physical and rehabilitation medicine. 2012;55(4):263-78.

14. Heneweer $H$, van Woudenberg $N J$, van Genderen F, Vanhees L, Wittink $H$. Measuring psychosocial variables in patients with (sub) acute low back pain complaints, at risk for chronicity: a validation study of the Acute Low Back Pain Screening Questionnaire-Dutch Language Version. Spine. 2010;35(4):447-52.

15. Fagundes FRC, Costa LOP, Fuhro FF, Manzoni ACT, de Oliveira NTB, Cabral CMN. Örebro Questionnaire: short and long forms of the Brazilian-Portuguese version. Quality of Life Research. 2015;24(11):2777-88.

16. The United Nations. Official Languages 2019, Mar 18 [Available from: http://www.un.org/en/sections/about-un/official-languages/.

17. Beaton DE, Bombardier C, Guillemin F, Ferraz MB. Guidelines for the process of cross-cultural adaptation of self-report measures. Spine. 2000;25(24):3186-91.

18. Terwee CB, Bot SD, de Boer MR, van der Windt DA, Knol DL, Dekker J, et al. Quality criteria were proposed for measurement properties of health status questionnaires. Journal of clinical epidemiology. 2007;60(1):34-42. 
19. Algarni AS, Ghorbel S, Jones JG, Guermazi M. Validation of an Arabic version of the Oswestry index in Saudi Arabia. Annals of physical and rehabilitation medicine. 2014;57(9-10):653-63.

20. Alanazi F, Gleeson P, Olson S, Roddey T. Translation and Validation of the Arabic Version of the FearAvoidance Beliefs Questionnaire in Patients With Low Back Pain. Spine. 2017;42(7):E411-e6.

21. Terkawi AS, Sullivan M, Abolkhair A, Al-Zhahrani T, Terkawi RS, Alasfar EM, et al. Development and validation of Arabic version of the pain catastrophizing scale. Saudi journal of anaesthesia. 2017;11(Suppl 1):S63-s70.

22. Bier JD, Ostelo R, van Hooff ML, Koes BW, Verhagen AP. Validity and Reproducibility of the STarT Back Tool (Dutch Version) in Patients With Low Back Pain in Primary Care Settings. Physical therapy. 2017;97(5):561-70.

23. Gabel CP, Melloh M, Yelland M, Burkett B, Roiko A. Predictive ability of a modified Örebro Musculoskeletal Pain Questionnaire in an acute/subacute low back pain working population. European Spine Journal. 2011;20(3):449-57.

24. Linton SJ, Nicholas M, MacDonald S. Development of a short form of the Örebro Musculoskeletal Pain Screening Questionnaire. Spine. 2011;36(22):1891-5.

25. van Tulder M, Becker A, Bekkering T, Breen A, del Real MTG, Hutchinson A, et al. Chapter 3European guidelines for the management ofacute nonspecific low back painin primary care. European Spine Journal. 2006;15(2):s169-s91.

26. Delitto A, George SZ, Van Dillen LR, Whitman JM, Sowa G, Shekelle P, et al. Low back pain. The Journal of orthopaedic and sports physical therapy. 2012;42(4):A1-57.

\section{Supplementary Files}

This is a list of supplementary files associated with this preprint. Click to download.

- Appendix1ArabicOMPQ.pdf 